J. DIFFERENTIAL GEOMETRY

83 (2009) 663-691

\title{
TWISTED CONSTANT SCALAR CURVATURE KÄHLER METRICS AND KÄHLER SLOPE STABILITY
}

\author{
JACOPO STOPPA
}

\begin{abstract}
On a compact Kähler manifold we introduce a cohomological obstruction to the solvability of the constant scalar curvature ( $\mathrm{cscK}$ ) equation twisted by a semipositive form, appearing in works of Fine and Song-Tian.

As a special case we find an obstruction for a manifold to be the base of a holomorphic submersion carrying a cscK metric in certain "adiabatic" classes. We apply this to find new examples of general type threefolds with classes which do not admit a cscK representative.

When the twist vanishes our obstruction extends the slope stability of Ross-Thomas to effective divisors on a Kähler manifold. Thus we find examples of non-projective slope unstable manifolds.
\end{abstract}

\section{Introduction}

One of the central open problems in Kähler geometry is to characterise the Kähler classes represented by a constant scalar curvature Kähler (cscK) metric (or more generally an extremal metric).

Not much is known about this question in general except of course for the Calabi-Yau Theorem [31] and the well known work of Yau, Tian and others on Del Pezzo surfaces.

In the algebraic case of representing the first Chern class of an ample line bundle the question is related to stability in algebraic geometry by a conjecture of Yau [24], Tian [7] and Donaldson [10].

It is a fundamental result in the theory that the existence of a $\operatorname{cscK}$ metric in the algebraic case implies K-semistability in the sense of Donaldson (the shortest proof is given in [11]).

From this Ross-Thomas derived a cohomological obstruction to solving the cscK equation in the algebraic case known as slope stability for polarised manifolds [22]. This proved to be a very effective tool in the study of cscK metrics on projective bundles and algebraic surfaces, see e.g. $[\mathbf{2 1}]$.

Received 05.26.2008. 
In this paper we expand from this starting point in two directions.

Firstly with Theorem 1.6 we extend slope stability (with respect to effective divisors) to any Kähler class on a compact Kähler manifold, confirming a conjecture of Ross-Thomas. This is explained in 1.3 below. Non-projective examples are given in 5.13.

Our methods are necessarily different from those of Ross-Thomas, in particular they are differential-geometric in nature. The key fact in this connection is the lower bound for the K-energy proved by Donaldson [12] and in more generality Chen-Tian [3], see 1.6 below. Then Section 3 is devoted to showing that the slope stability condition with respect to an effective divisor arises precisely by imposing that this lower bound holds as the Kähler form concentrates along the divisor to some extent dictated by positivity.

The other main theme of this paper is that Kähler slope stability generalises to an equation introduced by Fine and which we call the twisted cscK equation. Solutions to the twisted cscK equation arise as zeros of the moment map for an action of the group of exact symplectomorphisms. This was observed by G. Székelyhidi in analogy to the case of $\csc$ metrics where it was shown by Donaldson $[\mathbf{8}]$ and Fujiki. The proof is given in Section 2 .

Thus our main obstruction result, Theorem 1.3, is stated in this more general setup.

This extension is not a mere formality however, as obstructing the twisted cscK equation leads to interesting geometric applications through the so-called "adiabatic limit" construction.

We hope to make this clear in the rest of this Introduction, see in particular the obstruction Theorem 1.5 and the application to general type threefolds explained in 1.4 below.

Notation. Throughout the paper we mix the additive and multiplicative notation for divisors and line bundles. We write $\sqrt{-1}$ for the complex number $i$ and $d^{c}$ for the operator $-\frac{\sqrt{-1}}{2}(\partial-\bar{\partial})\left(\right.$ so $\left.d d^{c}=\sqrt{-1} \partial \bar{\partial}\right)$.

1.1. The twisted cscK equation. Let $M$ be a compact Kähler manifold with Kähler class $\Omega$ and let $\alpha$ be a closed pointwise semi-positive $(1,1)$-form on $M$. The equation that we are interested in, and which we call the twisted cscK equation is finding a metric $\omega \in \Omega$ such that

$$
S(\omega)-\Lambda_{\omega} \alpha=\widehat{S}_{\alpha}
$$

Here $S(\omega)$ is the scalar curvature, $\Lambda_{\omega} \alpha$ denotes the trace of $\alpha$ with respect to $\omega$

$$
\Lambda_{\omega} \alpha \omega^{n}=n \alpha \wedge \omega^{n-1}
$$


and $\widehat{S}_{\alpha}$ is the only possible topological constant, given by

$$
\widehat{S}_{\alpha}=\frac{n\left(c_{1}(X)-[\alpha]\right) \cup[\omega]^{n-1}}{[\omega]^{n}} .
$$

In particular if $\alpha=0$ we recover the cscK equation,

$$
S(\omega)=\widehat{S},
$$

and $\widehat{S}$ is just the average scalar curvature.

Equation (1.1) is a generalisation of (1.2) which arises naturally in the work of Fine [13], [14] and Song-Tian [26], [27]. There twisted cscK metrics in dimension $n$ are related to genuine $\operatorname{cscK}$ metrics in higher dimension by a limiting process.

Let $\pi: M \rightarrow B$ be a holomorphic submersion of Kähler manifolds endowed with a relatively ample line bundle $L$. The so-called adiabatic classes on $M$ are given by

$$
\Omega_{r}=c_{1}(L)+r \pi^{*} \Omega_{B}
$$

where $\Omega_{B}$ is any Kähler class on $B$. These are certainly Kähler for large $r$.

Suppose that (1.2) can be solved in all the fibres of $\pi$ inside the restriction of $c_{1}(L)$, and that the fibres and base carry no nontrivial holomorphic vector fields. In this case Fine proved that (1.2) can be solved in $\Omega_{r}$ for $r \gg 0$ provided that the twisted equation (1.1) is solvable in $\Omega_{B}$ with respect to a special choice of $\alpha$. Loosely speaking the relevant $\alpha$ is the pullback of the Weil-Petersson type metric from the moduli space of cscK metrics on a fibre.

We take up the application to adiabatic cscK metrics in Section 4. In particular Theorem 1.5 gives a necessary numerical condition for their existence.

In a different vein Song-Tian studied the untraced form of (1.1) (see also $[\mathbf{1 4}])$,

$$
\operatorname{Ric}(\omega)=\lambda \omega+\alpha
$$

in connection with the Kähler-Ricci flow on an elliptic surface. In this case $\lambda=-1$ and $\alpha$ is given by the pullback of the genuine Weil-Petersson metric plus a singular contribution corresponding to multiple fibres.

They prove that the Kähler-Ricci flow converges to a solution of (1.4) on the base which they call a generalised Kähler-Einstein metric.

In a recent preprint $[\mathbf{2 7}]$ this result is extended to the general case of a projective manifold with positive Kodaira dimension and semiample canonical bundle. The limiting solutions still have the form (1.4).

1.2. Obstructions. Our first result gives a cohomological obstruction for the twisted cscK equation to admit a solution in the class $\Omega$.

Recall that the positive cone

$$
\mathcal{P} \subset H^{1,1}(M, \mathbb{C}) \cap H^{2}(M, \mathbb{R})
$$


is given by cohomology classes $\Theta$ which evaluate positively on irreducible subvarieties, that is

$$
\int_{V} \Theta^{p}>0
$$

for any $p$-dimensional irreducible analytic subvariety $V \subset M$.

Let $D \subset M$ be an effective divisor.

Definition 1.1. The Seshadri constant of $D$ with respect to $\Omega$ is given by

$$
\epsilon(D, \Omega)=\sup \left\{x: \Omega-x c_{1}(\mathcal{O}(D)) \in \mathcal{P}\right\} .
$$

We define coefficients $\alpha_{i}, i=0,1$ by

$$
\begin{aligned}
& \alpha_{1}(x)=\frac{\int_{X} c_{1}(D) \cup\left(\Omega-x c_{1}(D)\right)^{n-1}}{(n-1) !} \\
& \alpha_{2}(x)=\frac{\int_{X} c_{1}(D) \cup\left(c_{1}(X)-[\alpha]-c_{1}(D)\right) \cup\left(\Omega-x c_{1}(D)\right)^{n-2}}{2(n-2) !} .
\end{aligned}
$$

Definition 1.2. The twisted Ross-Thomas polynomial of $(X, \Omega)$ with respect to $D$ and $\alpha$ is given by

$$
\mathcal{F}_{\alpha}(\lambda)=\int_{0}^{\lambda}(\lambda-x) \alpha_{2}(x) d x+\frac{\lambda}{2} \alpha_{1}(0)-\frac{\widehat{S}_{\alpha}}{2} \int_{0}^{\lambda}(\lambda-x) \alpha_{1}(x) d x .
$$

Theorem 1.3 (Stability condition). If (1.1) is solvable in the class $\Omega$ then

$$
\mathcal{F}_{\alpha}(\lambda) \geq 0
$$

for all effective divisors $D \subset X$ and $0 \leq \lambda \leq \epsilon(D, \Omega)$.

The proof will be given in Section 3.

Remark 1.4. For $\lambda<\varepsilon(D, \Omega)$ we have $\alpha_{1}(x)>0$ so the quotient

$$
\mu_{\lambda}\left(\mathcal{O}_{D}, \Omega\right)=\frac{\int_{0}^{\lambda}(\lambda-x) \alpha_{2}(x) d x+\frac{\lambda}{2} \alpha_{1}(0)}{\int_{0}^{\lambda}(\lambda-x) \alpha_{1}(x) d x}
$$

is well defined. Following Ross-Thomas we call this slope. Then the inequality (1.7) can be rewritten as

$$
\mu_{\lambda}\left(\mathcal{O}_{D}, \Omega\right) \geq \frac{\widehat{S}_{\alpha}}{2}
$$

The point is that choosing $\Omega=c_{1}(L), \alpha=0$ gives back the slope stability condition of Ross-Thomas [22] in the algebraic setting.

The relation of our work with slope stability is explained in 1.3 below.

Next we obtain an obstruction to the existence of the adiabatic cscK holomorphic submersions introduced above. 
Theorem 1.5. Let $\pi: M \rightarrow B$ be a holomorphic submersion endowed with a relatively ample line bundle $L$, which is fibrewise cscK with fibrewise average scalar curvature $S_{b}$. If the adiabatic classes (1.3) admit cscK metrics for $r \gg 0$ then (1.7) holds with

$$
[\alpha]=c_{1}\left(\pi_{*} K_{X \mid B}\right)+\frac{S_{b}}{n+1} c_{1}\left(\pi_{*} L\right)
$$

for all effective divisors $D \subset B$ and $0 \leq \lambda \leq \epsilon\left(D, \Omega_{B}\right)$.

An analogous statement holds when $L$ is replaced by a class $\Omega_{0}$ which is positive and cscK along the fibres. The proof will be given in Section 4 .

We will give concrete examples of how one can apply both results in Section 5. In particular Corollary 5.3 gives an a priori obstruction for an algebraic surface with a fixed Kähler class to be the base of an adiabatic cscK submersion.

1.3. Kähler slope stability. Ross-Thomas prove that subschemes can give an obstruction to the solvability of the cscK equation in the projective case $\Omega=c_{1}(L)$. Given a subscheme $Z \subset M$ they define a cohomological slope $\mu_{\lambda}\left(\mathcal{O}_{Z}, L\right)$ depending on a positive parameter $\lambda$ less than the Seshadri constant. They prove that if $(1.2)$ is solvable in $c_{1}(L)$ then the slope inequality

$$
\mu_{\lambda}\left(\mathcal{O}_{Z}, L\right) \geq \mu(M, L)
$$

where $\mu(M, L)=\frac{-n K_{M} \cdot L^{n-1}}{2 L^{n}}$ must hold for all $Z \subset M$ and $0<\lambda \leq$ $\epsilon(Z, L)$. In other words $(M, L)$ must be slope semistable. The particular form of (1.10) is of course modelled on slope stability for vector bundles (in terms of quotient sheaves).

As we already observed in the special case of effective divisors (1.10) is equivalent to inequality (1.7) with the choices $\Omega=c_{1}(L), \alpha=0$.

Motivated by the Uhlenbeck-Yau Theorem for vector bundles [29] Ross-Thomas extended the definition of slope to any analytic subvariety of a Kähler manifold, and conjectured that (1.10) still gives a necessary condition for (1.2) to be solvable. We refer to [22] Section 4.4 for more details. In our case we only need to allow any Kähler class $\Omega$ in place of $c_{1}(L)$.

Thus Theorem 1.3 with the choice $\alpha=0$ gives a proof of this conjecture in the case of effective divisors, namely

Theorem 1.6. If a Kähler class $\Omega$ on a Kähler manifold $M$ admits a cscK representative it is slope semistable with respect to effective divisors.

A concrete non-projective example is given in Lemma 5.13. 
Remark 1.7. The case of divisors is somehow central in slope stability. In general blowing up the subscheme $Z \subset M$ reduces to the exceptional divisor $E \subset \mathrm{Bl}_{Z} M$, polarised by $L^{1 / \lambda} \otimes \mathcal{O}(-E)$. This trick is not directly relevant to us because for our methods we need smoothness of the ambient space. However one might hope to keep track of the resolution of singularities in this context. Since all our concrete examples are based on effective divisors, we do not pursue this here.

In a different vein Ross-Panov [21] Section 4 prove that for algebraic surfaces it is enough to check slope stability with respect to divisors.

1.4. General type 3-folds. By the Calabi-Yau Theorem if $K_{M}$ is ample we can find a Kähler-Einstein metric in its first Chern class. On the other hand one may ask what happens for classes far from $c_{1}\left(K_{M}\right)$. These have proved hard to obstruct and the first example of a surface with ample canonical bundle and a class with no cscK representative was found only in recent years by Ross [18].

To date the only examples for general type threefolds are somewhat trivial, namely products of an obstructed surface with a curve of genus at least 2 , endowed with the product class. In 5.6, 5.8, 5.10 we apply our results on adiabatic cscK metrics to provide new examples of general type threefolds with obstructed classes.

1.5. Log-geometry. Given an effective divisor $D$ on $M$, log-geometry replaces the canonical bundle $K_{M}$ by $K_{M}+D$ and calls $D$ the boundary of $M$. Note that this is precisely what happens when we replace slope stability with our condition (1.8), that is we replace $c_{1}\left(K_{M}\right)$ by $c_{1}\left(K_{M}\right)+[\alpha]$ throughout. On the other hand Székelyhidi [28] has conjectured that the slope stability condition for the existence of a complete extremal metric on the complement of a reduced effective divisor $D$ is precisely log-slope, replacing $c_{1}\left(K_{M}\right)$ by $c_{1}\left(K_{M}+\mathcal{O}(D)\right)$ in the slope inequality. Thus in geometric terms coupling with an adiabatic class in a holomorphic submersion and removing a divisor should have the same slope stability condition.

1.6. K-energy. Finally a few words about our method of proof. Our results are based on the computation of a suitable energy functional, the "twisted K-energy", along a metric degeneration breaking the Kähler form into currents of integration (see Section 3 and Theorem 3.1 in particular).

Thus we introduce the natural analogue of the K-energy on the space of Kähler metrics in a given Kähler class. For any $\phi \in \mathcal{H}$, the space of Kähler potentials of $\omega$, let $\omega_{\phi}=\omega+d d^{c} \phi, \mu_{\phi}=\frac{\omega_{\phi}^{n}}{n !}$.

Definition 1.8. The variation of the twisted K-energy at $\omega_{\phi}$ is the 1 -form

$$
\delta \mathcal{M}_{\alpha}(\delta \phi)=-\int_{M} \delta \phi\left(S\left(\omega_{\phi}\right)-\Lambda_{\omega_{\phi}} \alpha-\widehat{S}_{\alpha}\right) \mu_{\phi}
$$


for $\delta \phi \in T_{\phi} \mathcal{H} \cong C^{\infty}(M, \mathbb{R})$.

The form $\delta \mathcal{M}_{\alpha}$ on $\mathcal{H}$ is closed. Choosing a base-point $\omega \in \Omega$ and integrating along any path gives a well-defined twisted $K$-energy $\mathcal{M}_{\alpha}$.

This is just the sum of the well known K-energy [16] and a variant of the J-functional introduced by Chen [2]. Its critical points are precisely solutions of (1.1). It is not hard to prove that these are actually local minimisers.

Since it is the sum of two functionals which are convex along geodesics in $\mathcal{H}, \mathcal{M}_{\alpha}$ is also convex. More precisely the second derivative along a path $\phi=\phi_{t}$ is given by

$$
\begin{aligned}
\frac{d^{2}}{d t^{2}} \mathcal{M}_{\alpha} & =\left\|\bar{\partial} \nabla^{(1,0)} \dot{\phi}\right\|_{\phi}^{2}+(\partial \dot{\phi} \wedge \bar{\partial} \dot{\phi}, \alpha)_{\phi} \\
& -\int_{M}\left(\ddot{\phi}-\frac{1}{2}\left|\nabla^{1,0} \dot{\phi}\right|_{\phi}^{2}\right)\left(S\left(\omega_{\phi}\right)-\Lambda_{\omega_{\phi}} \alpha-\widehat{S}_{\alpha}\right) \mu_{\phi}
\end{aligned}
$$

where all the metric quantities are computed with respect to $\omega+d d^{c} \phi$. The geodesic equation is $\ddot{\phi}-\frac{1}{2}\left|\nabla^{1,0} \dot{\phi}\right|_{\phi}^{2}=0$. Semmes and Donaldson have shown that this is the equation of geodesics for a negatively curved locally symmetric space structure on $\mathcal{H}$.

If either $\alpha$ is strictly positive at a point or $M$ has no Hamiltonian holomorphic vector fields, $\mathcal{M}_{\alpha}$ is strictly convex along geodesics in $\mathcal{H}$. In this case, the results of Chen-Tian [3] on the regularity of weak geodesics imply uniqueness of solutions of (1.1). In general, they imply the following lower bound on $\mathcal{M}_{\alpha}$.

Lemma 1.9. If (1.1) is solvable the twisted $K$-energy $\mathcal{M}_{\alpha}$ is bounded below in $\Omega$.

Acknowledgements. This work took shape mainly thanks to conversations with G. Székelyhidi. The moment map interpretation in particular is due to him. I thank my advisor R. Thomas for his great support and for suggesting to prove Theorem 1.6 via the K-energy. J. Fine, A. Ghigi, D. Panov, S. Rollenske and J. Ross provided useful comments and suggestions. Referee corrections improved the original manuscript essentially. I am grateful to the Geometry Groups at Pavia (in particular G. Pirola) and Imperial College, and especially to S. K. Donaldson for many interesting discussions about cscK metrics and the material presented here.

\section{Moment map interpretation}

In this section we show that when the form $\alpha$ is symplectic the operator

$$
\omega \mapsto \widehat{S}_{\alpha}-S(\omega)+\Lambda_{\omega} \alpha
$$


can be viewed as a moment map, essentially by combining Donaldson's moment map computations in [8] and [9]. In particular this holds when $\alpha$ is positive (rather than only semipositive as in the rest of the paper).

Let $(B, \omega)$ be a symplectic manifold of dimension $n$ and let us assume for simplicity that $H^{1}(B)=0$. We write $\mathscr{J}$ for the space of integrable complex structures on $B$ which are compactible with $\omega$. The space $\mathscr{J}$ admits a natural symplectic form, and the action of the group of symplectomorphisms $\operatorname{Symp}(B, \omega)$ preserves this symplectic form. Let us identify the Lie algebra of $\operatorname{Symp}(B, \omega)$ with $C_{0}^{\infty}(B, \mathbb{R})$, the smooth functions with vanishing integral, via the Hamiltonian construction. Also, using the $L^{2}$ inner product with respect to the volume form $\frac{\omega^{n}}{n !}$ let us identify the dual of $C_{0}^{\infty}(B, \mathbb{R})$ with itself.

Theorem 2.1 (Donaldson). The map

$$
J \mapsto \hat{S}-S(J),
$$

is an equivariant moment map for the action of $\operatorname{Symp}(B, \omega)$ on $\mathscr{J}$. Here $S(J)$ is the scalar curvature of the Kähler metric given by $(\omega, J)$ and $\hat{S}$ is its average which is independent of $J$.

Now let $M$ be diffeomorphic to $B$ and fix a symplectic form $\alpha$ on $M$. Let $\mathscr{M}$ be the space of diffeomorphisms $f: B \rightarrow M$ in a fixed homotopy class. The tangent space $T_{f} \mathscr{M}$ to $\mathscr{M}$ at $f$ can be identified with the space of vector fields on $M$. This has a natural symplectic form given by

$$
\Omega_{f}(v, w)=\int_{B} f^{*}(\alpha(v, w)) \frac{\omega^{n}}{n !} .
$$

The group $\operatorname{Symp}(B, \omega)$ acts on $\mathscr{M}$ by composition on the right, preserving $\Omega$.

Theorem 2.2 (Donaldson). The map

$$
\nu: f \mapsto \Lambda_{\omega} f^{*}(\alpha)
$$

is an equivariant moment map for the action of $\operatorname{Symp}(B, \omega)$ on $\mathscr{M}$.

Proof. We give the simple proof for the reader's convenience. Let us first consider the infinitesimal action of $\operatorname{Symp}(B, \omega)$ on $\mathscr{M}$. For this let $H \in C^{\infty}(B)$, and write $X_{H}$ for the Hamiltonian vector field on $B$ generated by $H$. The infinitesimal action at a point $f \in \mathscr{M}$ is given by $f_{*}\left(X_{H}\right)$. To show that $\nu$ is a moment map we therefore have to show

$$
\left\langle d \nu_{f}(w), H\right\rangle=\Omega_{f}\left(f_{*} X_{H}, w\right)=\int_{S} f^{*}\left(\alpha\left(f_{*} X_{H}, w\right)\right) \frac{\omega^{n}}{n !},
$$

where $w \in T_{f} \mathscr{M}$.

Let $f_{t}$ be a path in $\mathscr{M}$ such that $f_{0}=f$ and $\frac{d}{d t} f_{t}=w$ at $t=0$. Then

$$
d \nu_{f}(w)=\left.\frac{d}{d t}\right|_{t=0} \Lambda_{\omega} f^{*}(\alpha)=\Lambda_{\omega} f^{*}\left(d\left(\iota_{w} \alpha\right)\right) .
$$


Therefore

$$
\begin{aligned}
\left\langle d \nu_{f}(w), H\right\rangle & =\int_{B} H d\left(f^{*}\left(\iota_{w} \alpha\right)\right) \wedge \frac{\omega^{n-1}}{(n-1) !} \\
& =\int_{B} f^{*}\left(\iota_{w} \alpha\right) \wedge \iota_{X_{H}} \omega \wedge \frac{\omega^{n-1}}{(n-1) !} \\
& =\int_{B} f^{*}\left(\alpha\left(f_{*} X_{H}, w\right)\right) \frac{\omega^{n}}{n !} .
\end{aligned}
$$

This shows that $\nu$ is a moment map.

q.e.d.

We now combine the above two moment maps. Let us fix a complex structure $I$ on $M$ which is compatible with $\alpha$ and consider the subspace of $\mathscr{M} \times \mathscr{J}$ given by

$$
\mathscr{S}=\left\{\left(f, f^{*}(I)\right) \mid f \in \mathscr{M} \text { such that } f^{*}(I) \text { is compatible with } \omega\right\} .
$$

Then $\mathscr{S}$ is preserved by the action of $\operatorname{Symp}(B, \omega)$, and the moment map restricted to $\mathscr{S}$ is given by

$$
\left(f, f^{*}(I)\right) \mapsto-S\left(\omega, f^{*}(I)\right)+\Lambda_{\omega} f^{*}(\alpha)+C,
$$

where $C$ is a constant such that the integral over $B$ is zero.

Following Donaldson let us now consider the complexification of the action of $\operatorname{Symp}(B, \omega)$. This can only be done on the level of the Lie algebras. Both $\mathscr{J}$ and $\mathscr{M}$ have natural complex structures so we can complexify the infinitesimal action of the Lie algebra $C_{0}^{\infty}(B)$. This gives rise to an integrable distribution on $\mathscr{M} \times \mathscr{J}$ which is tangent to $\mathscr{S}$. We think of the leaves of the resulting foliation of $\mathscr{S}$ as the complexified orbits.

If $\left(f, f^{*}(I)\right)$ is a zero of the moment map, then

$$
S\left(\left(f^{-1}\right)^{*} \omega, I\right)-\Lambda_{\left(f^{-1}\right)^{*} \omega} \alpha
$$

is constant. If in addition $\left(f, f^{*}(I)\right)$ is in the complexified orbit of (id, $I$ ) then in fact $\left(f^{-1}\right)^{*} \omega$ is in the same Kähler class as $\omega$, so we have a solution of the twisted cscK equation.

\section{Proof of the stability condition}

As already mentioned in the Introduction what we actually prove in this section is a result on the asymptotic behaviour of the twisted K-energy.

Theorem 3.1. Let $D \subset M$ be an effective divisor. For any basepoint $\omega \in \Omega$ and $0<\lambda<\epsilon(D, \Omega)$ there exists a family of Kähler forms $\omega_{\varepsilon} \in \Omega, 0<\varepsilon \leq 1$ with $\omega_{1}=\omega$ and such that as $\varepsilon \rightarrow 0$

$$
\mathcal{M}_{\alpha}\left(\omega_{\varepsilon}\right)=-\pi \mathcal{F}_{\alpha}(\lambda) \log (\varepsilon)+\text { l.o.t. }
$$

By Lemma 1.9 this settles Theorem 1.3. 
Remark 3.2. It must be pointed out that expansions for the $\mathrm{K}$ energy of the form (3.1) are known to hold in a different setup, namely for the pullback Fubini-Study metric along the fibres of a $\mathbb{C}^{*}$-action in projective space (under some regularity assumptions) by the work of Paul-Tian [19], Phong-Sturm-Ross [20] and others.

Theorem 3.1 will be proved in several steps. We first recall the fundamental Nakai-Moishezon criterion of Demailly-Paun [5].

Theorem 3.3 (Demailly-Paun). The Kähler cone $\mathcal{K}$ is a connected component of the positive cone $\mathcal{P}$.

Corollary 3.4. $\epsilon(D, \Omega)=\sup \left\{x: \Omega-x c_{1}(\mathcal{O}(D)) \in \mathcal{K}\right\}$.

This holds because $\Omega-x c_{1}(\mathcal{O}(D))$ is in the connected component of $\Omega \in \mathcal{K}$.

Let $\omega \in \Omega$ be any Kähler form, and $h$ be any Hermitian metric on $\mathcal{O}(D)$ with curvature form $\Theta$. We begin by defining the family in Theorem 3.1 near $\varepsilon=0$.

Given a canonical section $\sigma \in H^{0}(\mathcal{O}(D))$ we define potentials

$$
\psi_{\varepsilon}=\frac{1}{2} \log \left(\varepsilon^{2}+|\sigma|_{h}^{2}\right)
$$

for $0<\varepsilon \ll 1$. By the Poincaré-Lelong equation there is a weak convergence

$$
d d^{c} \lambda \psi_{\varepsilon} \rightarrow-\lambda \Theta+\lambda[D]
$$

where $[D]$ denotes the (closed, positive) current of integration along $D$. By Corollary 3.4 for $0<\lambda<\epsilon(D, \Omega)$ we can find a potential $u$ (independent of $\varepsilon$ ) such that

$$
\eta=\omega-\lambda \Theta+d d^{c} u>0 .
$$

We define our family for $0<\varepsilon \ll 1$ by

$$
\omega_{\varepsilon}=\omega+d d^{c} u+d d^{c} \lambda \psi_{\varepsilon} .
$$

Since $[D]$ is positive,

$$
\eta+\lambda[D]>\eta
$$

holds in the sense of currents, and the sequence $\omega_{\varepsilon}$ converges weakly to $\eta+\lambda[D]$, so the inequality

$$
\omega_{\varepsilon}>\eta
$$

holds in the sense of currents for $\varepsilon \ll 1$. In other words $\omega_{\varepsilon}-\eta$ is a strictly positive $(1,1)$-current for all small $\varepsilon$. But since it is also a smooth $(1,1)$ form, this implies it is actually a Kähler form (more generally a similar pointwise statement holds for a current with locally $L^{1}$ coefficients, see [4] Section 3).

We conclude that for $0<\varepsilon \ll 1, \omega_{\varepsilon}$ is a Kähler form. We still need to prescribe the base-point to be $\omega_{1}=\omega$. This can be achieved by choosing 
$\psi_{\varepsilon} \rightarrow 0$ as $\varepsilon \rightarrow 1$, and making $u=u_{\varepsilon}$ dependent on $\varepsilon$ away from $\varepsilon=0$ so that $u_{\varepsilon} \rightarrow 0$ as $\varepsilon \rightarrow 1$ and $\omega+d d^{c} \psi_{\varepsilon}+d d^{c} u_{\varepsilon}$ is always Kähler.

The next important observation is that we have a uniform $C^{\infty}$ bound for $\omega_{\varepsilon}$ away from $D$ (that is, in any compact subset of $X \backslash D$ ). This means that for the sake of proving Theorem 3.1 we need only study the behaviour of $\mathcal{M}_{\alpha}$ near points of $\operatorname{supp}(D)$.

Let us first write down in detail the model case of $x \in \operatorname{supp}(D)$ near which $D$ is reduced and smooth. This will be enough for the general case thanks to standard results in the theory of currents.

So choose coordinates $\left(z=\left\{z_{i}\right\}_{i=1}^{n-1}, w\right)$ near $x$ such that $D$ is locally given by $\{w=0\}$. Then

$$
\psi_{\varepsilon}=\frac{1}{2} \log \left(\varepsilon^{2}+e^{-2 \varphi}|w|^{2}\right)
$$

near $x$, where $e^{-\varphi}$ is the weight for $h$ on $\left.\mathcal{O}(D)\right|_{U}$ with respect to the Euclidean norm $|\cdot|$. We make the first order expansion in the $D$ transversal direction

$$
e^{-2 \varphi}=e^{-2 \widetilde{\varphi}}(1+g)
$$

where $\widetilde{\varphi}=\widetilde{\varphi}(z)$ does not depend on the transversal coordinate $w$ while $g=g(z, w)$. In other words $\widetilde{\varphi}$ is the restriction of $\varphi$ to $D$, extended to be constant in the $w$ direction. In particular $g$ and all its $z_{i}, \bar{z}_{j}$ derivatives vanish identically along $\{w=0\}$. We will use this fact repeatedly. Moreover from now on we assume that we have chosen normal $z$-coordinates at $x$ so that

$$
\widetilde{\varphi}=\partial_{z_{i}} \widetilde{\varphi}=\partial_{\bar{z}_{j}} \widetilde{\varphi}=0 \text {. }
$$

Since $\widetilde{\varphi}$ is independent of $w$ this holds in a small slice $\{z=z(x)\}$.

We will need local expressions for the derivatives of the potential $\psi_{\varepsilon}$.

Lemma 3.5. In the slice $\{z=z(x)\}$,

$$
\begin{aligned}
\partial_{\varepsilon} \psi_{\varepsilon} & =\frac{\varepsilon}{\varepsilon^{2}+|w|^{2}(1+g)}, \\
\partial_{w} \partial_{\bar{w}} \psi_{\varepsilon} & =\frac{\varepsilon^{2}\left(1+g+\bar{w} \partial_{\bar{w}} g+w \partial_{w} g+|w|^{2} \partial_{w} \partial_{\bar{w}} g\right)}{2\left(\varepsilon^{2}+|w|^{2}(1+g)\right)^{2}} \\
& +\frac{|w|^{4}\left(-\partial_{\bar{w}} g \partial_{w} g+(1+g) \partial_{w} \partial_{\bar{w}} g\right)}{2\left(\varepsilon^{2}+|w|^{2}(1+g)\right)^{2}}, \\
\partial_{z_{i}} \partial_{\bar{w}} \psi_{\varepsilon} & =\frac{\varepsilon^{2} w \partial_{z_{i}} g}{2\left(\varepsilon^{2}+|w|^{2}(1+g)\right)^{2}}-\frac{|w|^{4} \partial_{z_{i}} g \partial_{\bar{w}} g}{2\left(\varepsilon^{2}+|w|^{2}(1+g)\right)^{2}} \\
& +\frac{|w|^{2} \partial_{z_{i}} \partial_{\bar{w}} g}{2\left(\varepsilon^{2}+|w|^{2}(1+g)\right)}, \\
\partial_{z_{i}} \partial_{\bar{z}_{j}} \psi_{\varepsilon} & =\frac{|w|^{2}\left(-2(1+g) \partial_{z_{i}} \partial_{\bar{z}_{j}} \widetilde{\varphi}+\partial_{z_{i}} \partial_{\bar{z}_{j}} g\right)}{2\left(\varepsilon^{2}+|w|^{2}(1+g)\right)}-\frac{\partial_{z_{i}} g \partial_{\bar{z}_{j}} g|w|^{4}}{2\left(\varepsilon^{2}+|w|^{2}(1+g)\right)^{2}} .
\end{aligned}
$$


Proof. First

$$
\partial_{\varepsilon} \psi_{\varepsilon}=\frac{\varepsilon}{\varepsilon^{2}+e^{-2 \widetilde{\varphi}}|w|^{2}(1+g)}=\frac{\varepsilon}{\varepsilon^{2}+|w|^{2}(1+g)} .
$$

The other terms are more complicated. We compute

$$
\begin{aligned}
\partial_{w} \partial_{\bar{w}} \psi_{\varepsilon} & =\frac{e^{2 \widetilde{\varphi}} \varepsilon^{2}\left(1+g+\bar{w} \partial_{\bar{w}} g+w \partial_{w} g+|w|^{2} \partial_{w} \partial_{\bar{w}} g\right)}{2\left(e^{2 \widetilde{\varphi}} \varepsilon^{2}+|w|^{2}(1+g)\right)^{2}} \\
& +\frac{|w|^{4}\left(-\partial_{\bar{w}} g \partial_{w} g+(1+g) \partial_{w} \partial_{\bar{w}} g\right)}{2\left(e^{2 \widetilde{\varphi}} \varepsilon^{2}+|w|^{2}(1+g)\right)^{2}} .
\end{aligned}
$$

In our normal coordinates this equals

$$
\begin{gathered}
\frac{\varepsilon^{2}\left(1+g+\bar{w} \partial_{\bar{w}} g+w \partial_{w} g+|w|^{2} \partial_{w} \partial_{\bar{w}} g\right)}{2\left(\varepsilon^{2}+|w|^{2}(1+g)\right)^{2}} \\
+\frac{|w|^{4}\left(-\partial_{\bar{w}} g \partial_{w} g+(1+g) \partial_{w} \partial_{\bar{w}} g\right)}{2\left(\varepsilon^{2}+|w|^{2}(1+g)\right)^{2}} .
\end{gathered}
$$

Similarly

$$
\begin{gathered}
\partial_{z_{i}} \partial_{\bar{w}} \psi_{\varepsilon}=-\frac{e^{2 \widetilde{\varphi}} \varepsilon^{2} w\left(1+g+\bar{w} \partial_{\bar{w}} g\right) \partial_{z_{i}} \widetilde{\varphi}}{\left(e^{2 \widetilde{\varphi}} \varepsilon^{2}+|w|^{2}(1+g)\right)^{2}} \\
+\frac{\left(e^{2 \widetilde{\varphi}} \varepsilon^{2} w-|w|^{4} \partial_{\bar{w}} g\right) \partial_{z_{i}} g+\left(e^{2 \widetilde{\varphi}} \varepsilon^{2}|w|^{2}+|w|^{4}(1+g)\right) \partial_{z_{i}} \partial_{\bar{w}} g}{2\left(e^{2 \widetilde{\varphi}} \varepsilon^{2}+|w|^{2}(1+g)\right)^{2}} .
\end{gathered}
$$

Using normal coordinates this simplifies to

$$
\frac{\varepsilon^{2} w \partial_{z_{i}} g}{2\left(\varepsilon^{2}+|w|^{2}(1+g)\right)^{2}}-\frac{|w|^{4} \partial_{z_{i}} g \partial_{\bar{w}} g}{2\left(\varepsilon^{2}+|w|^{2}(1+g)\right)^{2}}+\frac{|w|^{2} \partial_{z_{i}} \partial_{\bar{w}} g}{2\left(\varepsilon^{2}+|w|^{2}(1+g)\right)} \text {. }
$$

Finally

$$
\begin{aligned}
\partial_{z_{i}} \partial_{\bar{z}_{j}} \psi_{\varepsilon} & =\frac{|w|^{2} e^{2 \widetilde{\varphi}} \varepsilon^{2}\left(-2(1+g) \partial_{z_{i}} \partial_{\bar{z}_{j}} \widetilde{\varphi}-2 \partial_{z_{i}} \widetilde{\varphi} \partial_{\bar{z}_{j}} g\right)}{2\left(e^{2 \widetilde{\varphi}} \varepsilon^{2}+|w|^{2}(1+g)\right)^{2}} \\
& +\frac{|w|^{2}\left(e^{2 \widetilde{\varphi}} \varepsilon^{2}\left(2 \partial_{\bar{z}_{j}} \widetilde{\varphi}\left(2(1+g) \partial_{z_{i}} \widetilde{\varphi}-\partial_{z_{i}} g\right)+\partial_{z_{i}} \partial_{\bar{z}_{j}} g\right)\right)}{2\left(e^{2 \widetilde{\varphi}} \varepsilon^{2}+|w|^{2}(1+g)\right)^{2}} \\
& +\frac{|w|^{4}\left(-2(1+g)^{2} \partial_{z_{i}} \partial_{\bar{z}_{j}} \widetilde{\varphi}-\partial_{z_{i}} g \partial_{\bar{z}_{j}} g+(1+g) \partial_{z_{i}} \partial_{\bar{z}_{j}} g\right)}{2\left(e^{2 \widetilde{\varphi}} \varepsilon^{2}+|w|^{2}(1+g)\right)^{2}}
\end{aligned}
$$

In normal coordinates this becomes

$$
\frac{|w|^{2}\left(-2(1+g) \partial_{z_{i}} \partial_{\bar{z}_{j}} \widetilde{\varphi}+\partial_{z_{i}} \partial_{\bar{z}_{j}} g\right)}{2\left(\varepsilon^{2}+|w|^{2}(1+g)\right)}-\frac{\partial_{z_{i}} g \partial_{\bar{z}_{j}} g|w|^{4}}{2\left(\varepsilon^{2}+|w|^{2}(1+g)\right)^{2}} .
$$

q.e.d. 
In the situation above, for $p \geq 0$ we write

$$
\left(d d^{c} \psi_{\varepsilon}\right)^{p}=\alpha_{p, \varepsilon}+\beta_{p, \varepsilon}
$$

where

$$
\begin{aligned}
& \alpha_{p, \varepsilon}=(\sqrt{-1})^{p}\left(\sum_{i, j} \partial_{z_{i}} \partial_{\bar{z}_{j}} \psi_{\varepsilon} d z_{i} \wedge d \bar{z}_{j}+\sum_{i} \partial_{z_{i}} \partial_{\bar{w}} \psi_{\varepsilon} d z_{i} \wedge d \bar{w}\right. \\
& \left.+\sum_{j} \partial_{\bar{z}_{j}} \partial_{w} \psi_{\varepsilon} d w \wedge d \bar{z}_{j}\right)^{p} \\
& \beta_{p, \varepsilon}=(\sqrt{-1})^{p-1} p\left(\sum_{i, j} \partial_{z_{i}} \partial_{\bar{z}_{j}} \psi_{\varepsilon} d z_{i} \wedge d \bar{z}_{j}\right)^{p-1} \wedge\left(\partial_{w} \partial_{\bar{w}} \psi_{\varepsilon} \sqrt{-1} d w \wedge d \bar{w}\right)
\end{aligned}
$$

(setting $\beta_{0, \varepsilon}=0$ ). Then isolating the terms in Lemma 3.5 which are unbounded as $\varepsilon \rightarrow 0$ we find

Lemma 3.6. For $p \geq 2$

$$
\left(d d^{c} \psi_{\varepsilon}\right)^{p}=\alpha_{p, \varepsilon}+\beta_{p, \varepsilon}
$$

where

$$
\begin{gathered}
\alpha_{p, \varepsilon}=a^{i} \wedge \frac{\varepsilon^{2} w \partial_{z_{i}} g}{2\left(\varepsilon^{2}+|w|^{2}(1+g)\right)^{2}} d z_{i} \wedge d \bar{w} \\
+b^{\bar{j}} \wedge \frac{\varepsilon^{2} \bar{w} \partial_{\bar{z}_{i}} g}{2\left(\varepsilon^{2}+|w|^{2}(1+g)\right)^{2}} d \bar{z}_{j} \wedge d w \\
+c^{i \bar{j}} \wedge \frac{\varepsilon^{4}|w|^{2} \partial_{z_{i}} g \partial_{\bar{z}_{j}} g}{2\left(\varepsilon^{2}+|w|^{2}(1+g)\right)^{4}} d z_{i} \wedge d \bar{z}_{j} \wedge d w \wedge d \bar{w}+d^{i \bar{j}} \wedge d z_{i} \wedge d \bar{z}_{j}
\end{gathered}
$$

and $a^{i}, b^{\bar{j}}, c^{i \bar{j}}, d^{i \bar{j}}$ are uniformly bounded forms (as $\varepsilon \rightarrow 0$ ), while

$$
\begin{gathered}
\beta_{p, \varepsilon}=(\sqrt{-1})^{p-1} p\left(\sum_{i, j} \partial_{z_{i}} \partial_{\bar{z}_{j}} \psi_{\varepsilon} d z_{i} \wedge d \bar{z}_{j}\right)^{p-1} \\
\wedge \frac{\varepsilon^{2}\left(1+g+\bar{w} \partial_{\bar{w}} g+w \partial_{w} g+|w|^{2} \partial_{w} \partial_{\bar{w}} g\right)}{2\left(\varepsilon^{2}+|w|^{2}(1+g)\right)^{2}} \sqrt{-1} d w \wedge d \bar{w}+f
\end{gathered}
$$

and the form $f$ is uniformly bounded (as $\varepsilon \rightarrow 0$ ).

The cases $p=0,1$ on the other hand are trivial. When computing the K-energy we need to test the forms $\left(d d^{c} \psi_{\varepsilon}\right)^{p}$ against the function $\varepsilon \partial_{\varepsilon} \psi_{\varepsilon}$. 
Proposition 3.7. In the sense of currents on $M$,

$$
\varepsilon \partial_{\varepsilon} \psi_{\varepsilon} \rightarrow 0
$$

and, for $p \geq 1$,

$$
\varepsilon \partial_{\varepsilon} \psi_{\varepsilon}\left(d d^{c} \psi_{\varepsilon}\right)^{p} \rightarrow \frac{(-1)^{p+1} \pi}{2(1+p)} \Theta^{p-1} \wedge[D] .
$$

Proof. Weak convergence can be checked locally. Consider first the model case of a point $x \in \operatorname{supp}(D)$ near which $\operatorname{Supp}(D)$ is smooth. By taking a finite ramified cover we can assume that $D$ is reduced. This does not affect weak convergence. Choosing coordinates at $x$ as in Lemma 3.6 we know that

$$
\varepsilon \partial_{\varepsilon} \psi_{\varepsilon}=\frac{\varepsilon^{2}}{\varepsilon^{2}+|w|^{2}(1+g)}
$$

in a slice $\{z=z(z)\}$. The right hand side is uniformly bounded and it converges to 0 uniformly away from $w=0$, thus it converges to 0 weakly as $\varepsilon \rightarrow 0$. Thus (3.3) holds away from the set of singular points of $\operatorname{Supp}(D)$.

Weak convergence extends to all $M$ thanks to the Support and Skoda theorems, as in the proof of Lemma 2.1 of [5] for example.

The second weak convergence is more complicated. As for (3.3) it is enough to check it near a smooth, reduced point. Thus we use the decomposition in Lemma 3.6, and we first show

$$
\varepsilon \partial_{\varepsilon} \psi_{\varepsilon} \alpha_{p, \varepsilon} \rightarrow 0 .
$$

Since $\varepsilon \partial_{\varepsilon} \psi_{\varepsilon} \rightarrow 0$, multiplying by it kills all the bounded forms (in the $\varepsilon \rightarrow 0$ limit). By Lemma 3.6 the only unbounded terms appearing in $\alpha_{p, \varepsilon}$ are bounded forms wedged with

$$
\frac{\varepsilon^{2} w \partial_{z_{i}} g}{2\left(\varepsilon^{2}+|w|^{2}(1+g)\right)^{2}} d z_{i} \wedge d \bar{w}
$$

(or its conjugate), or

$$
\frac{\varepsilon^{4}|w|^{2} \partial_{z_{i}} g \partial_{\bar{z}_{j}} g}{2\left(\varepsilon^{2}+|w|^{2}(1+g)\right)^{4}} d z_{i} \wedge d \bar{z}_{j} \wedge d w \wedge d \bar{w} .
$$

Changing variable by $w=\varepsilon w^{\prime}$ to compare with a sequence of forms defined for $w^{\prime} \in \mathbb{C}$ and using Lebesgue's dominated convergence one can check that the forms

$$
\frac{\varepsilon^{2} w}{2\left(\varepsilon^{2}+|w|^{2}(1+g)\right)^{2}} d z_{i} \wedge d \bar{w}, \frac{\varepsilon^{4}|w|^{2}}{2\left(\varepsilon^{2}+|w|^{2}(1+g)\right)^{4}} d z_{i} \wedge d \bar{z}_{j} \wedge d w \wedge d \bar{w}
$$

converge to currents supported at $w=0$. In $\alpha_{p, \varepsilon}$, these forms are multiplied by $\partial_{z_{i}} g, \partial_{z_{i}} g \partial_{\bar{z}_{j}} g$ respectively. Both functions vanish for $w=0$. 
Thus the weak limit of the unbounded forms in $\alpha_{p, \varepsilon}$ is 0 , and multiplying by the uniformly bounded function $\varepsilon \partial_{\varepsilon} \psi_{\varepsilon}$ does not change this.

Finally we show that

$$
\varepsilon \partial_{\varepsilon} \psi_{\varepsilon} \beta_{p, \varepsilon} \rightarrow \frac{(-1)^{p+1} \pi}{2(1+p)} \Theta^{p-1} \wedge[D] .
$$

As for $\alpha_{p, \varepsilon}$ multiplying by $\varepsilon \partial_{\varepsilon} \psi_{\varepsilon}$ kills all bounded terms, and by Lemma 3.6 the unbounded part of $\beta_{p, \varepsilon}$ may be written as $(\sqrt{-1})^{p-1}$ times by

$$
\begin{aligned}
& p\left(\sum_{i, j} \frac{|w|^{2}\left(-2(1+g) \partial_{z_{i}} \partial_{\bar{z}_{j}} \widetilde{\varphi}+\partial_{z_{i}} \partial_{\bar{z}_{j}} g\right)}{2\left(\varepsilon^{2}+|w|^{2}(1+g)\right)} d z_{i} \wedge d \bar{z}_{j}\right. \\
& \left.-\frac{\partial_{z_{i}} g \partial_{\bar{z}_{j}} g|w|^{4}}{2\left(\varepsilon^{2}+|w|^{2}(1+g)\right)^{2}} d z_{i} \wedge d \bar{z}_{j}\right)^{p-1} \\
& \wedge \frac{\varepsilon^{2}\left(1+g+\bar{w} \partial_{\bar{w}} g+w \partial_{w} g+|w|^{2} \partial_{w} \partial_{\bar{w}} g\right)}{2\left(\varepsilon^{2}+|w|^{2}(1+g)\right)^{2}} d w \wedge d \bar{w} .
\end{aligned}
$$

The first factor is uniformly bounded, while the form

$$
\frac{\varepsilon^{2}\left(1+g+\bar{w} \partial_{\bar{w}} g+w \partial_{w} g+|w|^{2} \partial_{w} \partial_{\bar{w}} g\right)}{2\left(\varepsilon^{2}+|w|^{2}(1+g)\right)^{2}} d w \wedge d \bar{w}
$$

converges weakly to a current supported on $w=0$. In particular wedging by it kills (in the $\varepsilon \rightarrow 0$ limit) all bounded terms divisible by $z_{i}, z_{\bar{j}}$ derivatives of $g$, since they vanish along $\{w=0\}$. We are left to compute the weak limit of

$$
\begin{gathered}
\varepsilon \partial_{\varepsilon} \psi_{\varepsilon}(\sqrt{-1})^{p-1} p\left(\sum_{i, j} \frac{|w|^{2}\left(-2(1+g) \partial_{z_{i}} \partial_{\bar{z}_{j}} \widetilde{\varphi}\right)}{2\left(\varepsilon^{2}+|w|^{2}(1+g)\right)} d z_{i} \wedge d \bar{z}_{j}\right)^{p-1} \\
\wedge \frac{\varepsilon^{2}\left(1+g+\bar{w} \partial_{\bar{w}} g+w \partial_{w} g+|w|^{2} \partial_{w} \partial_{\bar{w}} g\right)}{2\left(\varepsilon^{2}+|w|^{2}(1+g)\right)^{2}} \sqrt{-1} d w \wedge d \bar{w} .
\end{gathered}
$$

We rewrite this as

$$
\begin{gathered}
\frac{(-1)^{p+1}}{2} p\left(d d^{c} \widetilde{\varphi}\right)^{p-1} \wedge \sqrt{-1} d w \wedge d \bar{w} . \\
\frac{\varepsilon^{4}(1+g)^{p-1}|w|^{2(p-1)}}{\left(\varepsilon^{2}+|w|^{2}(1+g)\right)^{p+2}}\left(1+g+\bar{w} \partial_{\bar{w}} g+w \partial_{w} g+|w|^{2} \partial_{w} \partial_{\bar{w}} g\right) .
\end{gathered}
$$

The second factor in this form is converging uniformly to 0 away from $\{w=0\}$. Moreover the change of variable $w=\varepsilon w^{\prime}$ together with dominated convergence show that the $w$-transversal integrals of this form are converging to

$$
\int_{0}^{2 \pi} \int_{\mathbb{R}} \frac{s^{2 p-1}}{\left(1+s^{2}\right)^{p+2}} d s d \theta=\frac{\pi}{p(p+1)},
$$


so that the form is converging in the sense of currents to

$$
\frac{\pi}{p(p+1)}[\{w=0\}] \text {. }
$$

Thus the required weak limit is

$$
\frac{(-1)^{p+1} \pi}{2(1+p)}\left(d d^{c} \widetilde{\varphi}\right)^{p-1} \wedge[\{w=0\}] .
$$

To finish the proof note that $d d^{c} \widetilde{\varphi}$ represents the restriction of the curvature form $\left.\Theta\right|_{D}$ (extended to be constant in the vertical direction) while $[\{w=0\}]$ represents $D$.

q.e.d.

We will need a well known integration by parts formula (see e.g. [2]), adapted to our situation.

\section{Lemma 3.8.}

$$
\delta \mathcal{M}_{\alpha}=\delta \int_{M} \log \left(\frac{\omega_{\phi}^{n}}{\omega^{n}}\right) \frac{\omega_{\phi}^{n}}{n !}+\widehat{S}_{\alpha} \delta I-\delta J_{\alpha},
$$

where

$$
\begin{gathered}
\delta I=\int_{M} \delta \phi \frac{\omega_{\phi}^{n}}{n !} \\
\delta J_{\alpha}=\int_{M} \delta \phi(\operatorname{Ric}(\omega)-\alpha) \wedge \frac{\omega_{\phi}^{n-1}}{(n-1) !} .
\end{gathered}
$$

Thanks to this formula the proof of Theorem 3.1 can be divided into three separate statements.

We first prove an expansion for the $J_{\alpha}$-functional.

Definition 3.9. We introduce the cohomological quantity, depending on a positive parameter $\lambda$,

$\mathcal{F}_{J_{\alpha}}(\lambda)=\sum_{p=1}^{n-1} \frac{(-1)^{p+1} \lambda^{p+1}}{2(p+1) !(n-1-p) !} \int_{M}\left([\alpha]-c_{1}(M)\right) \cup \Omega^{n-1-p} \cup c_{1}^{p}(\mathcal{O}(D))$.

Lemma 3.10.

$$
J_{\alpha}\left(\omega_{\varepsilon}\right)=-\pi \mathcal{F}_{J_{\alpha}}(\lambda) \log (\varepsilon)+\text { l.o.t. }
$$

Proof. We will actually find the limit of $\varepsilon \partial_{\varepsilon} J_{\alpha}$ as $\varepsilon \rightarrow 0$. The integrand for this functional is

$$
(n-1) !^{-1} \varepsilon \partial_{\varepsilon} \lambda \psi_{\varepsilon}(\operatorname{Ric}(\omega)-\alpha) \wedge \omega_{\varepsilon}^{n-1}
$$

which by the binomial theorem applied to $(1,1)$ forms can be rewritten as

$(n-1) !^{-1}(\operatorname{Ric}(\omega)-\alpha) \wedge \sum_{p=0}^{n-1}\left(\begin{array}{c}n-1 \\ p\end{array}\right)\left(\omega+d d^{c} u\right)^{n-1-p} \wedge \lambda^{p+1} \varepsilon \partial_{\varepsilon} \psi_{\varepsilon}\left(d d^{c} \psi_{\varepsilon}\right)^{p}$. 
By Corollary 3.7 this converges weakly to

$$
\begin{gathered}
\pi(n-1) !^{-1} \sum_{p=1}^{n-1}\left(\begin{array}{c}
n-1 \\
p
\end{array}\right) \frac{(-1)^{p+1} \lambda^{p+1}}{2(p+1)} \\
\cdot(\operatorname{Ric}(\omega)-\alpha) \wedge\left(\omega+d d^{c} u\right)^{n-1-p} \wedge \Theta^{p-1} \wedge[D] .
\end{gathered}
$$

Integrating over $M$ proves our claim.

q.e.d.

An identical argument applies to the $I$ functional.

Definition 3.11. We introduce the cohomological quantity

$$
\mathcal{F}_{I}(\lambda)=\sum_{p=1}^{n} \frac{(-1)^{p+1} \lambda^{p+1}}{2(p+1) !(n-p) !} \int_{M} \Omega^{n-p} \cup c_{1}^{p}(\mathcal{O}(D))
$$

\section{Lemma 3.12.}

$$
I\left(\omega_{\varepsilon}\right)=\pi \mathcal{F}_{I}(\lambda) \log (\varepsilon)+\text { l.o.t. }
$$

Finally we consider the $\int \log \operatorname{det}$ functional.

Definition 3.13. Define a cohomological quantity by

$$
\mathcal{F}_{\log }=\sum_{p=1}^{n} \frac{(-1)^{p-1} \lambda^{p}}{p !(n-p) !} \int_{M} \Omega^{n-p} \cup c_{1}(\mathcal{O}(D))^{p} .
$$

\section{Lemma 3.14.}

$$
\int_{M} \log \left(\frac{\omega_{\varepsilon}^{n}}{\omega^{n}}\right) \frac{\omega_{\varepsilon}^{n}}{n !}=-\pi \mathcal{F}_{\log } \log (\varepsilon)+\text { l.o.t. }
$$

Proof. We study the weak limit as $\varepsilon \rightarrow 0$ of the sequence of forms on $M$

$$
\log (\varepsilon)^{-1} \log \left(\frac{\omega_{\varepsilon}^{n}}{\omega^{n}}\right) \frac{\omega_{\varepsilon}^{n}}{n !}
$$

Note that

$$
\begin{aligned}
\omega_{\varepsilon}^{n} & =\left(\omega+d d^{c} u+d d^{c} \lambda \psi_{\varepsilon}\right)^{n} \\
& =\sum_{k=0}^{n}\left(\begin{array}{l}
n \\
k
\end{array}\right) \lambda^{k}\left(\omega+d d^{c} u\right)^{n-k} \wedge\left(d d^{c} \psi_{\varepsilon}\right)^{k} .
\end{aligned}
$$

We can check weak convergence locally as in the proof of Proposition 3.7. Thus we can further decompose using the forms in Lemma 3.6, namely

$$
\omega_{\varepsilon}^{n}=\sum_{k=0}^{n}\left(\begin{array}{l}
n \\
k
\end{array}\right) \lambda^{k}\left(\omega+d d^{c} u\right)^{n-k} \wedge \alpha_{k, \varepsilon}+\sum_{k=1}^{n}\left(\begin{array}{l}
n \\
k
\end{array}\right) \lambda^{k}\left(\omega+d d^{c} u\right)^{n-k} \wedge \beta_{k, \varepsilon} .
$$


To analyse the weak limit we make the change of variable $w=\varepsilon w^{\prime}$ to compare with a sequence of forms defined for $w^{\prime} \in \mathbb{C}$. By using Lemma 3.6 we see

$$
\left.\sum_{k=0}^{n}\left(\begin{array}{l}
n \\
k
\end{array}\right) \lambda^{k}\left(\omega+d d^{c} u\right)^{n-k} \wedge \alpha_{k, \varepsilon}\right|_{w=\varepsilon w^{\prime}}=\varepsilon^{2} a(\varepsilon)+b(\varepsilon)
$$

where $a(\varepsilon)$ is uniformly bounded while $b(\varepsilon) \rightarrow 0$ uniformly as $\varepsilon \rightarrow 0$. By the proof of the same Lemma we also have

$$
\left.\sum_{k=1}^{n}\left(\begin{array}{l}
n \\
k
\end{array}\right) \lambda^{k}\left(\omega+d d^{c} u\right)^{n-k} \wedge \beta_{k, \varepsilon}\right|_{w=\varepsilon w^{\prime}} \leq c(\varepsilon) \sum_{k=1}^{n} \frac{s^{2 k-1}}{\left(1+s^{2}\right)^{k+1}} d s d \theta
$$

which is $c(\varepsilon) O\left(s^{-3}\right) d s d \theta$ in polar coordinates for the vertical direction, where $c(\varepsilon)$ is a uniformly bounded form.

It follows that, using polar coordinates,

$$
\left.\frac{\omega_{\varepsilon}^{n}}{\omega^{n}}\right|_{w=\varepsilon w^{\prime}}=\widetilde{a}(\varepsilon)+\frac{1}{\varepsilon^{2}}\left(\widetilde{b}(\varepsilon)+O\left(s^{-3}\right)\right)
$$

where $\widetilde{a}(\varepsilon)$ is uniformly positive and bounded and $\widetilde{b}(\varepsilon) \rightarrow 0$ uniformly as $\varepsilon \rightarrow 0$ (note that $a, b$ are forms while $\widetilde{a}, \widetilde{b}$ are functions). Thus

$$
\left.\log \left(\frac{\omega_{\varepsilon}^{n}}{\omega^{n}}\right)\right|_{w=\varepsilon w^{\prime}}=\log \left(\varepsilon^{2} \widetilde{a}(\varepsilon)+\widetilde{b}(\varepsilon)+O\left(s^{-3}\right)\right)-\log \left(\varepsilon^{2}\right),
$$

and

$$
\begin{aligned}
\left.\frac{1}{\log (\varepsilon)} \log \left(\frac{\omega_{\varepsilon}^{n}}{\omega^{n}}\right) \frac{\omega_{\varepsilon}^{n}}{n !}\right|_{w=\varepsilon w^{\prime}} & =\left.\frac{\log \left(\varepsilon^{2} \widetilde{a}(\varepsilon)+\widetilde{b}(\varepsilon)+O\left(s^{-3}\right)\right)}{\log (\varepsilon)} \frac{\omega_{\varepsilon}^{n}}{n !}\right|_{w=\varepsilon w^{\prime}} \\
& -\left.2 \frac{\omega_{\varepsilon}^{n}}{n !}\right|_{w=\varepsilon w^{\prime}} .
\end{aligned}
$$

In the notation above for the volume at $\varepsilon$, the first term has the form

$$
\frac{1}{n ! \log \varepsilon} \log \left(\varepsilon^{2} \widetilde{a}(\varepsilon)+\widetilde{b}(\varepsilon)+O\left(s^{-3}\right)\right)\left(\varepsilon^{2} a(\varepsilon)+b(\varepsilon)+O\left(s^{-3}\right) d s d \theta\right) .
$$

The integrals of

$$
\log \left(\varepsilon^{2} \widetilde{a}(\varepsilon)+\widetilde{b}(\varepsilon)+O\left(s^{-3}\right)\right)\left(\varepsilon^{2} a(\varepsilon)+b(\varepsilon)+O\left(s^{-3}\right) d s d \theta\right)
$$

in the vertical direction are uniformly bounded, thus the first term converges weakly to 0 .

The weak limit of the second term, namely $-\left.2 \frac{\omega_{\varepsilon}^{n}}{n !}\right|_{w=\varepsilon w^{\prime}}$, is computed exactly as for the integrands of the $I$ and $J_{\alpha}$ functionals. $\quad$ q.e.d.

Thanks to the integration by parts formula Lemma 3.8 our asymptotic expansions for the $I, J_{\alpha}$ and $\int \log$ det functionals imply the asymptotic expansion

$$
\mathcal{M}_{\alpha}\left(\omega_{\varepsilon}\right)=-\pi\left(\mathcal{F}_{\log }+\mathcal{F}_{J}-\widehat{S}_{\alpha} \mathcal{F}_{I}\right) \log (\varepsilon)+\text { l.o.t. }
$$


Now Theorem 3.1 follows since

$$
\mathcal{F}_{\alpha}=\mathcal{F}_{\log }+\mathcal{F}_{J}-\widehat{S}_{\alpha} \mathcal{F}_{I},
$$

which in turn follows from the identities

Lemma 3.15.

$$
\begin{aligned}
\int_{0}^{\lambda}(\lambda-x) \alpha_{2}(x) d x+\frac{\lambda}{2} \alpha_{1}(0) & =\mathcal{F}_{\log }(\lambda)+\mathcal{F}_{J_{\alpha}}(\lambda) \\
\int_{0}^{\lambda}(\lambda-x) \alpha_{1}(x) d x & =-2 \mathcal{F}_{I} .
\end{aligned}
$$

This is a lengthy check. It is fully performed in [22], proof of Theorem 5.2 in the case $\Omega=c_{1}(L)$. The extension to any Kähler class $\Omega$ is purely formal.

Remark 3.16. Our proof of Theorem 3.1 is a metric analogue of the deformation to the normal cone used by Ross-Thomas. In other words we compute the twisted K-energy along a sequence of metrics on $M$ which converge to the Fubini-Study metric on the fibres of the projective bundle $\mathbb{P}(\nu(D) \oplus \mathcal{O}) \rightarrow D$ locally after rescaling (at least near smooth, reduced points of $D)$.

\section{The K-energy of a holomorphic submersion}

In this Section we relate our obstruction for the twisted cscK equation

to the problem of constant scalar curvature on a holomorphic submersion, as described in the Introduction.

Let $\pi: M \rightarrow B$ be a holomorphic submersion. For the rest of this section $n$ denotes the relative dimension, while $m$ is the dimension of the base.

We suppose there is a class $\Omega_{0} \in H^{1,1}(M, \mathbb{C}) \cap H^{2}(M, \mathbb{R})$ whose restriction to any fibre $M_{b}$ is a Kähler class containing a cscK metric $\omega_{b}$, depending smoothly on $b$. Fitting together these forms gives a representative $\omega_{0} \in \Omega_{0}$ whose fibrewise restriction is cscK. By adding $d d^{c}$ of some function pulled back from the base we can ensure that $\omega_{0}$ is actually Kähler.

The fibrewise scalar curvature and volume are fixed constants $S_{b}$, $\operatorname{vol}\left(M_{b}\right)$ respectively.

Choosing a Kähler form $\omega_{B} \in \Omega_{B}$ on the base, we are interested in the existence of a cscK metric in the adiabatic classes $\Omega_{r}=\Omega_{0}+r \pi^{*} \Omega_{B}$, $r \gg 0$.

We describe the aforementioned result of Fine in more detail. Note that the forms $\omega_{b}$ give a metric on the vertical tangent bundle $V$ and so on the line $\operatorname{det}(V)$. Taking the curvature we get a $(1,1)$-form $F_{V}$ representing $c_{1}(V)$. We denote by $F_{V H}$ the purely horizontal component of $F_{V}$ with respect to the horizontal-vertical decomposition induced by 
$\omega_{0}$. Define a $(1,1)$-form $a$ by $F_{V H}=-\operatorname{vol}\left(M_{b}\right) a$. The choice of sign is to follow our convention that $\alpha$ (as defined below) is a semipositive rather then seminegative form. Taking the fibrewise average of $-F_{V H}$ with respect to $\omega_{0}$ yields a $(1,1)$-form $\alpha$ on the base, in other words

$$
\alpha_{b}=\int_{M_{b}} a \omega_{b}^{n}=\frac{\int_{M_{b}}-F_{V H} \omega_{b}^{n}}{\operatorname{vol}\left(M_{b}\right)}
$$

where the integrals are form-valued. Semipositivity of $\alpha$ is granted by the following result. See the Introduction to [14] for a detailed discussion.

Theorem 4.1 (Fujiki-Schumacher [15], Fine [14]). The form $\alpha$ is semipositive. If the fibres have no nontrivial holomorphic vector fields and the submersion does not induce an isotrivial fibration on any curve in $B$ the form $\alpha$ is strictly positive.

Fine assumes that that the fibres $M_{b}$ have no nontrivial holomorphic vector fields and that the equation

$$
S\left(\omega_{B}\right)-\Lambda_{\omega_{B}} \alpha=\widehat{S}_{\alpha}
$$

is solvable for a Kähler metric $\omega_{B} \in \Omega_{B}$ on the base. Moreover $\omega_{B}$ must admit no nontrivial cohomologous deformations which are still solutions. The conclusion is the existence of a cscK metric in all adiabatic classes $\Omega_{r}$ for $r \gg 0$.

Remark 4.2. It follows from our discussion of the K-energy in Section 1 that the condition about deformations of $\omega_{B}$ is certainly satisfied if either $B$ has no nontrivial holomorphic vector fields or $\alpha$ is strictly positive at a single point.

Note that it is not known if solving (4.1) is necessary for the existence of adiabatic cscK metrics. We expect this to be a very difficult question. We will use instead the K-energy to provide an obstruction.

The variation of the twisted K-energy in this case is best written in the form

$$
\delta \mathcal{M}_{\alpha}(\phi)=-\int_{B} \delta \phi\left(S\left(\omega_{B, \phi}\right)-\Lambda_{\omega_{B, \phi}} \alpha-\widehat{S}_{\alpha}\right) \frac{\omega_{B, \phi}^{m}}{m !}
$$

where $\omega_{B, \phi}=\omega_{B}+d d^{c} \phi$.

We will show that $\operatorname{vol}\left(M_{b}\right) \mathcal{M}_{\alpha}$ is the leading order term when we expand the genuine $\mathrm{K}$-energy $\mathcal{M}$ on $M$ in the adiabatic limit. Thus if the adiabatic classes admit a cscK representative the twisted K-energy on the base must be bounded below. In turn Theorem 3.1 gives an obstruction to the existence of these metrics. More precisely, let

$$
\omega_{r}=\omega_{0}+r \omega_{B}
$$


We are concerned with the variation of the K-energy on $M$ with respect to

$$
\delta \omega_{r}=\delta\left(\omega_{0}+r \omega_{B, \phi}\right)=r d d^{c} \delta \phi
$$

that is

$$
\delta \mathcal{M}=-\int_{M} r \delta \phi\left(S\left(\omega_{r}\right)-\widehat{S\left(\omega_{r}\right)}\right) \omega_{r}^{n+m} .
$$

\section{Lemma 4.3.}

$$
\delta \mathcal{M}=\operatorname{vol}\left(M_{b}\right) \delta \mathcal{M}_{\alpha} r^{m}+O\left(r^{m-1}\right) .
$$

Corollary 4.4. If the class $\Omega_{r}$ admits a cscK representative for $r \gg 0$ then the twisted $K$-energy $\mathcal{M}_{\alpha}$ is bounded below in the class $\Omega_{B}$ on the base.

An expansion for the K-energy along an adiabatic submersion may be also found in $[\mathbf{2 7}]$ Section 6 . However since that does not quite cover our case we prefer to give a proof. We need a preliminary computation of scalar curvature. This is implicit in $[\mathbf{1 3}]$ Theorem 8.1 , but we need to write it down in full for our purposes.

Introduce the vertical Laplacian $\Delta_{V}$, characterised by

$$
\Delta_{V} u \omega_{b}^{n}=n\left(d d^{c} u\right)_{V V} \wedge \omega_{b}^{n-1},
$$

where $\left(d d^{c} u\right)_{V V}$ denotes the purely vertical component. The Kähler form $\omega_{0}$ on $M$ gives rise to a horizontal-vertical decomposition of forms; in particular

$$
\omega_{0}=\omega_{b}+\omega_{H}
$$

\section{Lemma 4.5.}

$$
S\left(\omega_{r}\right)=S\left(\omega_{b}\right)+r^{-1}\left(S\left(\omega_{B}\right)-\operatorname{vol}\left(M_{b}\right) \Lambda_{\omega_{B}} a+\Delta_{V}\left(\Lambda_{\omega_{B}} \omega_{H}\right)\right)+O\left(r^{-2}\right) .
$$

Proof. We need to compute the Ricci form of $\omega_{r}$. By the exact sequence of holomorphic vector bundles

$$
0 \rightarrow V \rightarrow T M \rightarrow H \rightarrow 0
$$

this is the sum of the curvatures of the induced metrics on the line bundles $\operatorname{det}(V), \operatorname{det}(H)$, say $F_{V}, F_{H}$. By definition of $a$

$$
F_{V}=\operatorname{Ric}\left(\omega_{b}\right)-\operatorname{vol}\left(M_{b}\right) a .
$$

For $\operatorname{det}(H)$, we get

$$
\begin{aligned}
F_{H} & =d d^{c} \log \left(\omega_{H}+r \omega_{B}\right)^{m} \\
& =d d^{c} \log \omega_{B}^{m}\left(1+r^{-1} m \frac{\omega_{H} \wedge \omega_{B}^{m-1}}{\omega_{B}^{m}}+O\left(r^{-2}\right)\right) \\
& =\operatorname{Ric}\left(\omega_{B}\right)+d d^{c} \log \left(1+r^{-1} \Lambda_{\omega_{B}} \omega_{H}+O\left(r^{-2}\right)\right) \\
& =\operatorname{Ric}\left(\omega_{B}\right)+r^{-1} d d^{c} \Lambda_{\omega_{B}} \omega_{H}+O\left(r^{-2}\right) .
\end{aligned}
$$


Taking traces,

$$
\begin{aligned}
\Lambda_{\omega_{r}} F_{V} & =S\left(\omega_{b}\right)-m \frac{\operatorname{vol}\left(M_{b}\right) a \wedge\left(\omega_{H}+r \omega_{B}\right)^{m-1}}{\left(\omega_{H}+r \omega_{B}\right)^{m}} \\
& =S\left(\omega_{b}\right)-m \frac{r^{m-1} \operatorname{vol}\left(M_{b}\right) a \wedge \omega_{B}^{m-1}+O\left(r^{m-2}\right)}{r^{m} \omega_{B}^{m}+O\left(r^{m-1}\right)} \\
& =S\left(\omega_{b}\right)-r^{-1} \operatorname{vol}\left(M_{b}\right) \Lambda_{\omega_{B}} a+O\left(r^{-2}\right), \\
\Lambda_{\omega_{r}} F_{H}= & m \frac{\operatorname{Ric}\left(\omega_{B}\right) \wedge\left(\omega_{H}+r \omega_{B}\right)^{m-1}}{\left(\omega_{H}+r \omega_{B}\right)^{m}} \\
+ & r^{-1} \Delta_{V} \Lambda_{\omega_{B}} \omega_{H}+m \frac{d d^{c}\left(r^{-1} \Lambda_{\omega_{B}} \omega_{H}\right) \wedge\left(\omega_{H}+r \omega_{B}\right)^{m-1}}{\left(\omega_{H}+r \omega_{B}\right)^{m}} \\
= & r^{-1}\left(S\left(\omega_{B}\right)+\Delta V \Lambda_{\omega_{B}} \omega_{H}\right)+O\left(r^{-2}\right) .
\end{aligned}
$$

q.e.d.

Proof of Lemma 4.3. Note that

$$
\begin{aligned}
\pi_{*} \omega_{r}^{n+m} & =\operatorname{vol}\left(M_{b}\right)\left(\omega_{H}+r \omega_{B}\right)^{m} \\
& =\operatorname{vol}\left(M_{b}\right) r^{m} \omega_{B}^{m}+O\left(r^{m-1}\right), \\
-\operatorname{vol}\left(M_{b}\right) \pi_{*} \Lambda_{\omega_{B}} a \omega_{r}^{n+m} & =-\operatorname{vol}\left(M_{b}\right) \Lambda_{\omega_{B}} \alpha r^{m} \omega_{B}^{m}+O\left(r^{m-1}\right), \\
\pi_{*} \Delta_{V} \Lambda_{\omega_{B}}\left(\omega_{H}\right) \omega_{r}^{n+m} & =0 .
\end{aligned}
$$

As a consequence

$\pi_{*} S\left(\omega_{r}\right) \omega_{r}^{m+n}=\left(S_{b}+r^{-1}\left(S\left(\omega_{B}\right)-\Lambda_{\omega_{B}} \alpha\right)\right) \operatorname{vol}\left(M_{b}\right) r^{m} \omega_{B}^{m}+O\left(r^{m-2}\right)$.

The average of $S\left(\omega_{r}\right)$ can be computed using this pushforward. We find

$$
\begin{gathered}
S_{b}+r^{-1}\left(\widehat{S\left(\omega_{B}\right)}-\operatorname{vol}(B)^{-1} \int_{B} \Lambda_{\omega_{B}} \alpha \omega_{B}^{m}\right)+O\left(r^{-2}\right) \\
=S_{b}+r^{-1} \widehat{S}_{\alpha}+O\left(r^{-2}\right) .
\end{gathered}
$$

So we see that for the K-energy

$$
\begin{aligned}
\delta \mathcal{M} & =-\int_{B} \pi_{*}\left(r \delta \phi\left(S\left(\omega_{r}\right)-\widehat{S\left(\omega_{r}\right)}\right) \omega_{r}^{n+m}\right) \\
& =\int_{B}(r \delta \phi) r^{-1}\left(S\left(\omega_{B, \phi}\right)-\Lambda_{\omega_{B, \phi}} \alpha-\widehat{S}_{\alpha}\right)\left(\operatorname{vol}\left(M_{b}\right) r^{m} \omega_{B}^{m}+O\left(r^{m-1}\right)\right) \\
& =\operatorname{vol}\left(M_{b}\right) \delta \mathcal{M}_{\alpha} r^{m}+O\left(r^{m-1}\right) .
\end{aligned}
$$

q.e.d.

We are finally in a position to prove our obstruction to adiabatic cscK submersions, Theorem 1.5. 
Proof of Theorem 1.5. By Corollary 4.4 the result follows immediately from the asymptotics of the twisted K-energy, Theorem 3.1, once we know the cohomology class of $\alpha$. This is computed in the Lemma below.

q.e.d.

Lemma 4.6. Suppose $\Omega_{0}=c_{1}(L)$ for a relatively ample line bundle L. Then

$$
[\alpha]=c_{1}\left(\pi_{*} K_{M \mid B}\right)+\frac{S_{b}}{n+1} c_{1}\left(\pi_{*} L\right) .
$$

Proof. By definition

$$
\begin{aligned}
{[\alpha]=\left[\pi_{*}\left(a \omega_{0}^{n}\right)\right] } & =\operatorname{vol}\left(M_{b}\right)^{-1}\left[\pi_{*}\left(\operatorname{Ric}\left(\omega_{b}\right) \wedge \omega_{0}^{n}\right)-\pi_{*}\left(F_{V} \wedge \omega_{0}^{n}\right)\right] \\
= & \frac{S_{b}}{n+1} \pi_{*} c_{1}(L)+\pi_{*}\left(c_{1}\left(K_{M \mid B}\right)\right)
\end{aligned}
$$

and the pushforwards $\pi_{*} L, \pi_{*} K_{M \mid B}$ are locally free.

q.e.d.

\section{Examples}

In this Section we give some applications of our obstruction for the (twisted) cscK equation.

5.1. General type 3-folds. We begin with a simple adaptation of a result of Ross-Panov [21].

Recall that an effective divisor $D=\sum_{i} m_{i} D_{i}$ ( $D_{i}$ irreducible components) is exceptional if $\left[D_{i} . D_{j}\right]$ is negative definite. Let $p_{a}(D)$ be the arithmetic genus of $D$, given by $\chi\left(\mathcal{O}_{D}\right)=1-p_{a}(D)$.

Lemma 5.1. Let $M$ be an algebraic surface containing an exceptional divisor $D, \alpha$ a semi-positive form. If the inequality

$$
2 p_{a}(D)-2+D \cdot[\alpha]>0
$$

holds, there exists a Kähler class $\Omega$ for which $\mathcal{F}_{\alpha}(\lambda)<0$ for all small positive $\lambda$.

Proof. This is essentially the argument in loc. cit. Theorem 3.2. We adapt this to our case for the sake of completeness.

On surfaces the stability condition (1.7) in its slope formulation is best rewritten as

$$
\frac{3\left(2 \Omega \cdot D-\lambda\left(\left(K_{M}+[\alpha]\right) \cdot D+D^{2}\right)\right)}{2 \lambda\left(3 \Omega \cdot D-\lambda D^{2}\right)} \geq \frac{-\left(K_{X}+[\alpha]\right) \cdot \Omega}{\Omega^{2}}
$$

when $\Omega-\lambda D$ is a positive class. If we were allowed to choose $\Omega$ so that $\Omega . D=0$, by adjunction we could simplify this inequality to

$$
\frac{3\left(2 p_{a}(D)-2+D \cdot[\alpha]\right)}{2 \lambda D^{2}} \geq \frac{-\left(K_{X}+[\alpha]\right) \cdot \Omega}{\Omega^{2}} .
$$

Since $D^{2}<0$, whenever $2 p_{a}(D)-2+D \cdot[\alpha]>0$ the left hand side diverges to $-\infty$ as $\lambda \rightarrow 0$, thus violating (1.7). 
Of course this argument is not quite rigorous since $\Omega$ is positive. What we do instead is to construct a sequence $\Omega_{s}$ of Kähler classes degenerating to a big class $\Omega_{0}$ such that $\Omega_{0} . D=0$. Then (5.3) must hold by continuity, provided we also have a strictly positive lower bound on the Seshadri constants $\epsilon\left(\Omega_{s}, D\right)$ in order to be able to substitute some positive value for $\lambda$.

Let $K$ be any reference Kähler form. We claim there is a choice of strictly positive numbers $r_{i}$ such that the sequence

$$
\Omega_{s}=(1+s) K+\sum_{i} r_{i} D_{i}
$$

satisfies our requests.

The symmetric matrix $\left[D_{i} . D_{j}\right]$ is negative definite, so by diagonalising we see that we can find strictly positive numbers $r_{i}$ with

$$
\sum_{i} D_{i} . D_{j} r_{i}=-K . D_{j}
$$

for all $j$. This settles $\Omega_{0} \cdot D=0$.

The lower bound on $\epsilon\left(\Omega_{s}, D\right)$ is more delicate. If we make the extra assumption

$$
\text { D. } D_{i} \leq 0
$$

for all $i$, a lower bound is simply given by $\min _{i}\left\{\frac{r_{i}}{m_{i}}\right\}>0$. One can prove that the extra assumption causes no loss of generality for algebraic surfaces, see loc. cit. Corollary 3.4 for details.

Remark 5.2. This complication only arises if $D$ has more than one irreducible component. We can avoid it in any example when $D$ can be chosen irreducible, but not in our examples later on, since a key ingredient is to take finite coverings.

Finally $\Omega_{0}$ is big since it is obtained by adding an effective divisor to an ample divisor.

q.e.d.

Corollary 5.3. If an algebraic surface $M$ contains an exceptional divisor $D$ with arithmetic genus $p_{a}(D) \geq 2$ then there exists a class $\Omega$ for which $\mathcal{M}_{\alpha}$ is unbounded below for all $\alpha \geq 0$. If $p_{a}(D) \geq 1$ the same holds for all $\alpha>0$.

Thus in the first case we know a priori that $(M, \Omega)$ cannot be the base of an adiabatic cscK submersion.

We will show how this can be applied to construct classes which do not admit a cscK representative on some threefolds of general type. The idea is to start from a Kodaira surface $S$ with a suitable exceptional divisor $D$. By a result of Morita we obtain a threefold holomorphic submersion $X \rightarrow S^{\prime}$ upon taking a finite covering $S^{\prime} \rightarrow S$.

Theorem 5.4 (Morita [17]). Let $S$ be a surface with a holomorphic submersion to a smooth curve, with base and fibres of genus at least 2. 
There exists a finite covering $S^{\prime} \rightarrow S$ and a non-isotrivial holomorphic submersion $\mathcal{X} \rightarrow S^{\prime}$ whose fibres are curves of genus at least 2.

Remark 5.5. Fine [14], Section 4 uses Morita's result to give new examples of cscK metrics. We will do the converse, using it to obstruct some particular classes.

Then we use Corollary 5.3 and Corollary 4.4 to obstruct the adiabatic classes on $X$. Let us do a few cases in detail.

5.1.1. Let $C$ be a smooth curve of genus $g$ at least 2. Consider the product $S=C \times C$ with diagonal $\delta$ and fibre-classes $f_{1}, f_{2}$. S has ample canonical bundle $K_{S}=(2 g-2)\left(f_{1}+f_{2}\right)$ and $\delta$ is an exceptional divisor (of genus genus $g$ ) since $\delta^{2}=2-2 g$.

If $S^{\prime} \rightarrow S$ is any finite covering then $K_{S^{\prime}}>0$ and the preimage $\delta^{\prime}$ is again an exceptional divisor of genus at least 2 .

So choose a finite covering $S^{\prime} \rightarrow S$ as in 5.4 to get a 3 -fold $X \rightarrow S^{\prime}$. Fix any Kähler class $H$ on $S^{\prime}$. Define Kähler classes $\Omega_{s}=(1+s) H+$ $\sum_{i} r_{i} \delta_{i}$ as in the proof of 5.1 .

Lemma 5.6. The general type 3-fold $X$ admits no cscK metrics in the classes

$$
a c_{1}\left(K_{X \mid S^{\prime}}\right)+r \pi^{*} \Omega_{s}
$$

for $a \in \mathbb{R}^{+}, s \ll 1, r \gg 0$.

Remark 5.7. The original surface example of Ross $[\mathbf{1 8}]$ is in fact the self-product of a nongeneric curve $C$.

5.1.2. Let $C$ be a smooth curve with genus $g$ at least 2 and a free action of a group $G$ of order $d$ on it. This provides a finite $d$-fold covering $\pi: B \rightarrow C$. Let $\Sigma \subset B \times C$ be the union of the graphs of $g \circ \pi, g \in G$. It is well known that there exists a $d$-fold branched covering $\pi_{1}: S \rightarrow B \times C$, branched precisely along $\Sigma$, and that $S$ is smooth. The canonical bundle $K_{S}$ is ample, and the pre-image $\Gamma_{1}$ of the graph $\Gamma \subset B \times C$ of $\pi$ is an exceptional divisor of genus at least two, since $\Gamma^{2}=d(2-2 g)$. Moreover, the natural map $S \rightarrow C$ is a holomorphic submersion. This means that we can apply Morita's construction: there is a finite covering $\pi_{2}: S^{\prime} \rightarrow S$ such that $S^{\prime}$ is the base of a non-isotrivial holomorphic submersion $Y \rightarrow S^{\prime}$ whose fibres are smooth curves of genus at least 2. Again $K_{S^{\prime}}>0$ and the preimage $\Gamma_{2}$ of $\Gamma_{1}$ under $\pi_{2}$ is an exceptional divisor of genus at least 2. Setting $\Omega_{s}=(1+s) H+\sum_{i} r_{i} \Gamma_{2, i}$ as before (for any Kähler class $H$ on $S^{\prime}$ ) we find

Lemma 5.8. The general type 3-fold $Y$ admits no cscK metrics in the classes

$$
a c_{1}\left(K_{Y \mid S^{\prime}}\right)+r \pi^{*} \Omega_{s}
$$

for $a \in \mathbb{R}^{+}, s \ll 1, r \gg 0$. 
Remark 5.9. Before the general results of $[\mathbf{2 1}]$ obstructed classes on Atiyah-Hirzebruch surfaces had been constructed by Shu [25].

5.1.3. Another class of examples can be derived from a recent construction of Catanese-Rollenske [1]. Let $C$ be a smooth curve of genus $g$ at least 2 and choose a nonempty subset $\mathcal{S} \subset \operatorname{Aut}(C)$ (note that $\mathcal{S}$ is not necessarily a subgroup). An element $s \in \mathcal{S}$ determines the graph $\Gamma_{s} \subset S=C \times C$. Assume that $\Gamma_{s} \cap \Gamma_{t}=\emptyset$ for $s \neq t$; this translates into a group-theoretic condition in $\operatorname{Aut}(C)$ which holds for many choices. This disjoint union gives a divisor $D=\cup_{s \in \mathcal{S}} \Gamma_{s} \subset S$. Choose a fibre $F \subset S$ for the projection $p_{1}$, and fix the topological type of a ramified cover $F^{\prime} \rightarrow F$, that is a surjection $\psi: \pi_{1}(F \backslash D) \rightarrow \pi_{1}(F) \rightarrow 0$. Catanese-Rollenske prove that after a base change induced by a finite covering $f: C^{\prime} \rightarrow C$, there exists a ramified cover $r: S^{\prime} \rightarrow f^{*} S$, ramified exactly along $f^{*} D$, which is a Kodaira fibration for the projection $p_{1} \circ f \circ r$, and such that the class of the induced ramified cover of fibres is the prescribed $\psi$.

Now we take one more finite covering $\rho: S^{\prime \prime} \rightarrow S^{\prime}$ in order to apply Morita's Theorem and get a Kodaira fibration $Z \rightarrow S^{\prime \prime}$ with smooth curve fibres of genus at least 2. For all $t \in \mathcal{S}, \Gamma_{t}^{\prime \prime}=\rho^{*} f^{*} r^{*} \Gamma_{t}$ is an exceptional divisor of genus at least 2 . Write $\Gamma_{t, i}^{\prime \prime}$ for the irreducible components. For any Kähler class $H$ on $S^{\prime}$ we form the classes $\Omega_{s}=$ $(1+s) H+\sum_{i} r_{i} \Gamma_{t, i}^{\prime \prime}$ as in the previous examples.

Lemma 5.10. The general type 3-fold $Z$ admits no cscK metrics in the classes

$$
a c_{1}\left(K_{Z \mid S^{\prime \prime}}\right)+r \pi^{*} \Omega_{s}
$$

for $a \in \mathbb{R}^{+}, s \ll 1, r \gg 0$.

5.2. Slope unstable non-projective manifolds. One might try to prove Theorem 1.6 by a deformation argument. For example it is a classical result of Kodaira that any compact Kähler surface admits arbitrarily small deformations which are projective. If we could perturb the cscK metric at the same time this would give an alternative proof of 1.6 for surfaces.

This motivates us to look for a genuinely non-projective example in higher dimensions. This is based on Voisin's manifold, combined with the following result for projective bundles.

Lemma 5.11. Let $E \rightarrow B$ be a vector bundle on the Kähler manifold $\left(B, \Omega_{B}\right)$. Let $\mathcal{O}_{\mathbb{P}}(1)$ denote the relative hyperplane line bundle. If $E$ is Mumford-destabilised by a corank 1 subbundle $F \subset E$ then $\mathbb{P}(E)$ admits no cscK metrics in the classes

$$
\Omega_{r}=c_{1}\left(\mathcal{O}_{\mathbb{P}}(1)\right)+r \pi^{*} \Omega_{B}
$$

for $r \gg 0$. 
Proof. This is the Kähler version of a special case of [22] Theorem 5.12 .

Since $\mathbb{P}(F) \subset \mathbb{P}(E)$ is a divisor, all the computions in loc. cit. hold in $N E^{1}(\mathbb{P}(E))$ and therefore carry over without any change to our situation, with the care of replacing the line bundle $L^{\otimes r}$ there with the form $r \Omega_{B}$. Note in passing that a large part of the proof in loc. cit. is devoted to prove that $\epsilon\left(\mathbb{P}(F), \Omega_{r}\right)=1$ for $r \geq r_{0}$ where $r_{0}$ does not depend on $F$ (using boundedness of quotients). This is required to establish a general correspondence with Mumford-stability. However for our weak statement we only need to prove $\epsilon\left(\mathbb{P}(F), \Omega_{r}\right)=1$ for $r \geq r_{0}(F)$. This is trivial since $c_{1}\left(\mathcal{O}_{E}(1)\right)-x c_{1}\left(\mathcal{O}_{F}(1)\right)$ is relatively ample for $0<x<1$.

q.e.d.

Voisin [30] constructed Kähler manifolds in all dimensions $\geq 4$ which are not homotopy equivalent to projective ones. The simplest example in dimension 4 is obtained by a torus $T$ with an endomorphism $f: T \rightarrow T$. Consider the product $T \times T$ with projections $p_{i}, i=1,2$. There are four sub-tori in $T \times T$ given by the factors $T_{i}=p_{i}^{*} T$, the diagonal $T_{3}$ and the graph $T_{4}$ of $f$. Blow up the intersections of all $T_{k}$, that is a finite set $Q \subset T \times T$. Then blow up once more along the proper transforms of the $T_{k}$ 's. The result of this process is of course a Kähler manifold $M$. Voisin proves that for a special choice of $(T, f), M$ is not homotopy equivalent to (and so a fortiori not deformable to) a projective manifold.

For any $q \in Q$, let $E_{q}$ be the component of the exceptional divisor for

$$
\mathrm{Bl}_{Q}(T \times T) \rightarrow T \times T
$$

over $q \in Q$. We write $\mathcal{O}_{M}\left(-E_{q}\right)$ for the pullback of $\mathcal{O}\left(-E_{q}\right)$ to $M$. The rank 2 vector bundle $\mathcal{O}_{M}\left(-E_{q}\right) \oplus \mathcal{O}_{M}$ is Mumford-destabilised by the line bundle $\mathcal{O}_{M}\left(-E_{q}\right)$. Fix a Kähler class $\Omega_{M}$ on $M$. By Lemma 5.11 the projective bundle

$$
\pi: \mathbb{P}\left(\mathcal{O}\left(-E_{q}\right) \oplus \mathcal{O}_{M}\right) \rightarrow M
$$

admits no cscK metric in the classes $\mathcal{O}_{\mathbb{P}}(1)+r \pi^{*} \Omega_{M}$ for $r \gg 0$.

Note that by taking a projective bundle we have changed to homotopy type of $M$ so Voisin's Theorem does not immediately imply that $\mathbb{P}\left(\mathcal{O}\left(-E_{q}\right) \oplus \mathcal{O}_{M}\right)$ does not have projective deformations. However this is guaranteed by the following result on deformations of projective bundles $[6]$.

Theorem 5.12 (Demailly-Eckl-Peternell). Let $X$ be a compact complex manifold with a deformation $X \cong \mathcal{X}_{0} \hookrightarrow \mathcal{X} \rightarrow \Delta$. If $X=\mathbb{P}(E)$ for some vector bundle $E \rightarrow Y$ then $\mathcal{X}_{t}=\mathbb{P}\left(V_{t}\right)$ for some deformation $\mathcal{V}_{t} \rightarrow \mathcal{Y}_{t}$ 
Corollary 5.13. The Kähler manifold $X=\mathbb{P}\left(\mathcal{O}_{M}\left(-E_{q}\right) \oplus \mathcal{O}_{M}\right)$ is slope unstable (and so has no cscK metrics) with respect to the classes

$$
c_{1}\left(\mathcal{O}_{\mathbb{P}}(1)\right)+r \pi^{*} \Omega_{M}
$$

for $r \gg 0$. Moreover $X$ has no projective deformations.

\section{References}

[1] F. Catanese \& S. Rollenske, Double Kodaira fibrations. J. Reine Angew. Math. 628 (2009), 205-233, MR 2503241, Zbl pre05541584.

[2] X. Chen, On the lower bound of the Mabuchi energy and its application. Internat. Math. Res. Notices 2000, no. 12, 607-623, MR 1772078, Zbl 0980.58007.

[3] X. Chen \& G. Tian, Geometry of Kähler metrics and foliations by holomorphic discs. Publ. Math. Hautes Études Sci. No. 107 (2008), 1-107, MR 2434691.

[4] J.-P. Demailly, Courants positifs et thorie de l'intersection. Gaz. Math. 53 (1992) 131-159, MR 1175540, Zbl 0771.32010.

[5] J.-P. Demailly \& M. Paun, Numerical characterization of the Kähler cone of a compact Kähler manifold. Ann. of Math. (2) 159 (2004), no. 3, 1247-1274, MR 2113021, Zbl 1064.32019.

[6] J.-P. Demailly, T. Eckl \& T. Peternell, Line bundles on complex tori and a conjecture of Kodaira. Commentarii Math. Helvetici 80 (2005) 229-242, MR 2142242, Zbl 1078.32014.

[7] W.Y. Ding \& G. Tian, Kähler-Einstein metrics and the generalized Futaki invariant. Invent. Math. 110 (1992), no. 2, 315-335, MR 1185586, Zbl 0779.53044.

[8] S.K. Donaldson, Remarks on gauge theory, complex geometry and 4-manifold topology, Fields Medallists' Lectures (M. F. Atiyah and D. Iagolnitzer, eds.), World Sci. Publ., Singapore, 1997, pp.384-403, MR 1622931.

[9] S.K. Donaldson, Moment maps and diffeomorphisms. Surveys in differential geometry, 107-127, Surv. Differ. Geom., VII, Int. Press, Somerville, MA, 2000, MR 1919424, Zbl 1078.53084.

[10] S.K. Donaldson, Scalar curvature and stability of toric varieties. J. Differential Geom. 62 (2002), no. 2, 289-349, MR 1988506, Zbl 1074.53059.

[11] S.K. Donaldson, Lower bounds on the Calabi functional. J. Differential Geom. 70 (2005), no. 3, 453-472, MR 2192937, Zbl 1149.53042.

[12] S.K. Donaldson, Scalar curvature and projective embeddings. II. Q. J. Math. 56 (2005), no. 3, 345-356, MR 2161248, Zbl 1159.32012.

[13] J. Fine, Constant scalar curvature Kähler metrics on fibred complex surfaces. J. Differential Geom. 68 (2004), no. 3, 397-432, MR 2144537, Zbl 1085.53064.

[14] J. Fine, Fibrations with constant scalar curvature Kähler metrics and the CM-line bundle. Math. Res. Lett. 14 (2007), no. 2, 239-247, MR 2318622, Zbl 1132.53039 .

[15] A. Fujiki \& Schumacher, G. The moduli space of extremal compact Kähler manifolds and generalized Weil-Petersson metrics. Publ. Res. Inst. Math. Sci. 26 (1990), no. 1, 101-183, MR 1053910, Zbl 0714.32007.

[16] T. Mabuchi, K-energy maps integrating Futaki invariants, Tohoku Math. J. (2) 38 (1986), no. 4, 575-593, MR 0867064, Zbl 0619.53040. 
[17] S. Morita, Geometry of characteristic classes. Translations of Mathematical Monographs, 199. Iwanami Series in Modern Mathematics. American Mathematical Society, Providence, RI, 2001, MR 1826571, Zbl 0976.57026.

[18] J. Ross, Unstable products of smooth curves. Invent. Math. 165 (2006), no. 1, 153-162, MR 2221139, Zbl 1107.14011.

[19] S. Paul \& G. Tian, CM stability and the generalised Futaki invariant II. Preprint arXiv:math/0606505v2 [math.DG], to appear in Asterisque.

[20] D.H. Phong, J. Ross \& J. Sturm, Deligne pairings and the Knudsen-Mumford expansion. J. Differential Geom. 78 (2008), no. 3, 475-496, MR 2396251, Zbl 1138.14003.

[21] J. Ross \& D. Panov, Slope stability and exceptional divisors of high genus. Math. Ann. 343 (2009), no. 1, 79-101, MR 2448442, Zbl 1162.14034.

[22] J. Ross \& R.P. Thomas, An obstruction to the existence of constant scalar curvature Kähler metrics. J. Differential Geom. 72 (2006), 429-466, MR 2219940, Zbl 1125.53057 .

[23] J. Ross \& R.P. Thomas, A study of the Hilbert-Mumford criterion for the stability of projective varieties. J. Algebraic Geom. 16 (2007), no. 2, 201-255, MR 2274514, Zbl pre05161020.

[24] R. Schoen \& S.-T. Yau, Open problems section in Lectures on differential geometry. Conference Proceedings and Lecture Notes in Geometry and Topology, I. International Press, Cambridge, MA, 1994, MR 1333601, Zbl 0830.53001.

[25] Y. Shu, Unstable Kodaira Fibrations. Preprint arXiv:math/0612571v1 [math.DG].

[26] J. Song \& G. Tian, The Kähler-Ricci flow on surfaces of positive Kodaira dimension. Invent. Math. 170 (2007), no. 3, 609-653, MR 2357504.

[27] J. Song \& G. Tian, Canonical measures and Kähler-Ricci flow. Preprint arXiv:0802.2570v1 [math.DG].

[28] G. Székelyhidi, Extremal metrics and K-stability. Doctoral thesis, Imperial College, London (2006), available from arXiv:math/0611002v1 [math.DG].

[29] K. Uhlenbeck \& S.-T. Yau, On the existence of Hermitian-Yang-Mills connections in stable vector bundles. Comm. Pure Appl. Math. 39 (1986), 257-293, MR 0861491, Zbl 0615.58045.

[30] Voisin, C. On the homotopy types of compact Kähler and complex projective manifolds. Invent. Math. 157 (2004), no. 2, 329-343, MR 2076925, Zbl 1065.32010.

[31] S.-T. Yau, On the Ricci curvature of a compact Kähler manifold and the complex Monge-Ampére equation. I. Comm. Pure Appl. Math. 31 (1978), no. 3, 339-411, MR 0480350, Zbl 0369.53059.

C4 Great Court,

Trinity College

Cambridge CB2 1TQ

United KINGDOM

E-mail address: jacopostoppa@gmail.com 